\title{
A Self-Organized Resource Allocation Scheme for Decentralized Distributed Virtual Environments
}

\author{
Jean Botev \\ University of Luxembourg \\ L-1359 Luxembourg \\ Email: jean.botev@uni.lu
}

\author{
Ingo Scholtes \\ University of Trier \\ D-54286 Trier \\ Email: scholtes@syssoft.uni-trier.de
}

\begin{abstract}
With the growth of Massively Multiuser Virtual Environments (MMVEs) and increasingly interactive social networking platforms, it is widely accepted that their convergence renders today's centralized hosting approaches impracticable. To handle virtual environments of such massive scale, decentralized systems are necessary that also involve the resources of clients. The expedient design of techniques enabling this kind of next-generation decentralized distributed virtual environments (DDVEs) is a growing field of research. In the HyperVerse project, we aim at the provision of an infrastructure enabling such DDVEs, focusing on collaboration and self-organization as means to achieve a maximum degree of scalability.

In this paper we present a self-organized resource allocation scheme for DDVEs functioning independent of the underlying P2P network topology. Exploiting the heterogeneity of clients and utilizing locally available information only, it helps alleviate the load imposed by regions with a high user density as they often occur in such environments.

Evaluations show that both for the discovery of these regions and their alleviation the local views converge fast to a global one, with favorable effects on the overlay topology.
\end{abstract}

\section{INTRODUCTION}

Over the last decade a growing popularity of Massively Multiuser Virtual Environments (MMVEs) can be observed, be it social-centric online communities like for instance Second Life or game-centric environments, e.g. World of Warcraft, to name the largest. Particularly the idea of a three-dimensional Internet experience attracts much attention, and with the recent growth of social networking communities embracing interactive aspects, their convergence into some even larger, hybrid form becomes an easily understandable assumption.

It is widely accepted that ultimately the user numbers of a global-scale virtual environment of that kind render today's mostly centralized hosting approaches impractical and that they can only be handled on such a massive scale by decentralized - or at least hybrid systems - with a higher degree of commitment from the clients in terms of resources.

In the HyperVerse research project we aim at the provision of an infrastructure supporting such decentralized distributed virtual environments (DDVEs). The concept of a two-tier Peerto-Peer (P2P) architecture consisting of a highly structured backbone network of reliable, server-like machines and a loosely structured overlay on top, has been presented for instance in [1]. Clients are interconnected according to their virtual proximity and exchange data in a Torrent-like manner, forming a geometric overlay. It is an important objective to develop adaptive, self-organized techniques already on this level to unburden and support the backbone which is both responsible for neighbor discovery and the dynamic, fallback provision of data in the event of clients entering empty regions.

In this paper we present a self-organized resource allocation scheme that operates solely on local information to identify critical regions with a high user density in DDVEs, counteract appropriately and cushion effects that would lead to costly readjustment operations at backbone level. The core idea is to exploit the heterogeneity of peers and use the more powerful ones particularly in terms of bandwidth and reliability to facilitate the distribution of data by splitting off so-called virtual peers. By means of a gossip protocol, an aggregate gravitational field is created which influences the positions of these virtual peers. This way, a high responsiveness is ensured while at the same time the necessary inertia to prevent the mechanism from overreacting to fluctuations in population densities is provided.

The remainder of this paper is structured as follows: Section II gives a brief overview of the HyperVerse infrastructure, then focusing on aspects particularly relevant to the resource allocation scheme presented in the subsequent Section III. An evaluation of the scheme will be provided in Section IV. Before concluding, Section V features related work in the context of P2P overlay networks, load balancing and super-peer approaches for DDVEs. Finally, we summarize our contributions and outline perspectives in Section VI.

\section{Motivation And Model}

Before describing in more detail some core modeling aspects particularly relevant to our resource allocation scheme, we will give a brief overview of the HyperVerse project and its architecture in the following.

\section{A. HyperVerse}

Investigating fundamental principles suitable for the realization of extremely large-scale and highly interactive DDVEs, the HyperVerse project ${ }^{1}$ aims at creating a self-organizing and sustainable middleware service as a basis for future virtual environments like for instance a 3D Internet.

\footnotetext{
${ }^{1}$ http://hyperverse.syssoft.uni-trier.de
} 
Existing hosting approaches for MMVEs are still mostly based on centralized server infrastructures and therefore suffer from severe scalability issues when extended to a larger or even global scale. However, while there is consensus on the fact that $\mathrm{P} 2 \mathrm{P}$ technologies are particularly promising to enable DDVEs and a range of approaches presented in Section $\mathrm{V}$ exist, our approach differs in many key aspects also from them. One key feature of the HyperVerse middleware is the underlying two-tier P2P architecture depicted schematically in Figure 1: A loosely-coupled, geometric client overlay $T_{1}$ especially for real-time data distribution, as well as a federated and highly structured backbone network $T_{0}$ which explicitly includes client resources at its edges.

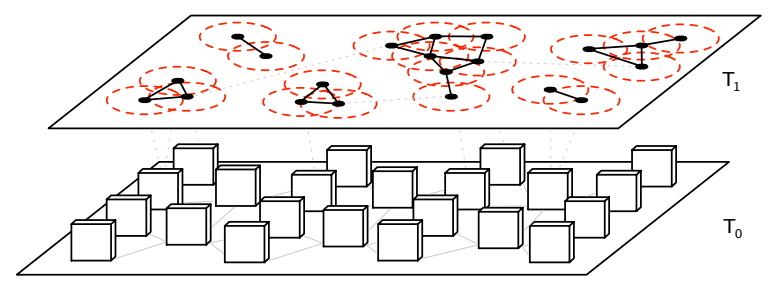

Fig. 1. Two-tier HyperVerse architecture.

Data dissemination in the HyperVerse is based on a Torrentlike technology [2], taking into account virtual geography and thus exploiting access locality. In order to provide the scalability needed to handle virtual environments of such large scale and dynamics, inter-client exchange of data is effected whenever possible. Clients are interconnected based on their proximity in virtual space via a geographic indexing service, effectuating specific qualities of the network, e.g. the power-law degree distribution, and allowing for self-adaptation of the statistical structure. By maintaining these direct links between nearby peers, costly routing mechanisms can be avoided as - due to the nature of virtual environments - most communication takes place in direct interaction or mutual visibility between peers and therefore is covered for most part by nearest neighbor connections. For more details on the backbone service as well as the neighbor discovery and data distribution mechanisms, we refer to [1].

The mobility and concentration of peers however will be addressed in more detail, as these are aspects with important implications on the functioning of the geometric overlay and hence also play a particularly decisive role in the design of our scheme.

\section{B. Hot Spot Regions in DDVEs}

The movement of avatars, i.e. instances representing users and their behavior in virtual environments, can differ from real-life motion patterns due to the possibility of fast travel, teleportation or in general the fluctuation of users known as churn. Still, a large number of users is likely to remain stable in terms of locality as many virtual environments focus on user-to-user interaction or longer activities (also involving user-to-object interaction) that take place in one specific area.
This collaborative aspect has the effect that motion will focus upon a specific set of locations, often with a self-energizing character, drawing in further users.

These regions can form in an extremely dynamic fashion or on the other hand also become very stable and static. Places like these are generally called hot spots, with their dynamic, burstlike variation being commonly known as flash crowds. A hot spot can be informally defined as a region within a virtual environment where a large number of users gather for a variety of possible reasons. Hot spots for instance can be landmarks of constant interest (e.g. a capital of a virtual region, sights etc.), or temporary interest (unique or spontaneous events like concerts, meeting places etc.).

Efficiently and timely alleviating traffic bursts in hot spot regions is one of the prime concerns in the design of technologies for DDVEs.

\section{SCHEME}

In this section, we present a distributed protocol for alleviating the hot spot problem in DDVEs. The scheme is based on the following assumptions: First of all, bandwidth and reliability of clients participating in the provision of DDVEs are highly heterogenous. As a consequence, apart from the visible representation of their user's avatar, more capable clients split off one (or more) invisible representations of their resources, so-called virtual peers. These then facilitate the distribution of object content and the dissemination of movement updates in a range around their virtual position. A gossip-based scheme is used to identify proximate hot spots while a virtual gravitational field built from purely local gossip-based information exchange guides the virtual peers to hot spot regions.

Prior to detailing the underlying protocol and algorithmic aspects, we will clarify some terminology and describe the related concepts of multiplexers and virtual peers.

\section{A. Multiplexers and Virtual Peers}

So far, only the concept of virtual peers has been mentioned explicitly. These are peers which equal the others in their responsibilities concerning data distribution. Instead of being controlled by a user's movement though, virtual peers are driven by an epidemic scheme (in which they also partake as equals) and gravitation, both described in detail in Sections III-B and III-C. The other key difference to the other peers lies in the fact that virtual peers are inducing preferential connections, i.e. if a virtual peer is in proximity, a peer is always rather connected to the virtual peer than to another peer. The motivation behind this preferential connect lies in the fact that virtual peers are provided by those peers with highest bandwidth and reliability.

We call these peers that split off and host one or more virtual peers multiplexers. They are selected by the backbone when joining the network by using a metric considering mainly bandwidth as well as taking into account previous session lengths. In certain $\mathrm{P} 2 \mathrm{P}$ systems, it has been shown that previous session lengths can be used as an estimator for 
future session lengths [3]. This way, the existing heterogeneity can be harnessed to improve overall system scalability and reliability. Due to the aforementioned two-tier structure of the HyperVerse, multiplexers can be identified without expensive collaborative calculation of a selection-parameter [4].

\section{B. Epidemic Hot Spot Detection}

The identification of hot spots in our scheme is based on an epidemic protocol. Such protocols have emerged as an efficient communication paradigm especially for large-scale and dynamic distributed systems, maintaining both simplicity and scalability at constant communication cost [5]. Generally, they function particularly well in network topologies with a good expansion [6] as exhibited also by the overlay we are operating on. The detection is based on an aggregate of peers' local perception of regional density and information exchange with a random neighbor is effected in periodic intervals.

For the internal prioritization of hot spots as well as automatic position updates of the virtual peers, we introduce a mass concept in analogy to physics. The mass of a hot spot is then defined by the amount of peers it contains:

Let $h_{i}$ be a hot spot region within the virtual environment containing $n$ peers. With $m_{1}, \ldots, m_{n}$ denoting the individual mass of each peer, we define the cumulative mass $M_{i}$ of the hot spot as

$$
M_{i}=\sum_{i=1}^{n} m_{i} .
$$

With $l_{1}, \ldots, l_{n}$ representing the location of the individual peers in virtual space, a hot spot $p_{i}$ 's center of mass $C_{i}$ is then defined as

$$
C_{i}=\frac{\sum_{j=1}^{n} l_{j} \cdot m_{j}}{M_{i}} .
$$

Each peer maintains a fixed-size local list $H_{i}$ of identified potential hot spot regions. Furthermore, each peer $p_{i}$ also defines a maximum lookahead distance $L_{i}$ that determines the range within which the query for hot spots is deducted.

The following algorithm describes the aggregation scheme in case that there is an information exchange between two peers $p_{i}$ and $p_{j}$ :

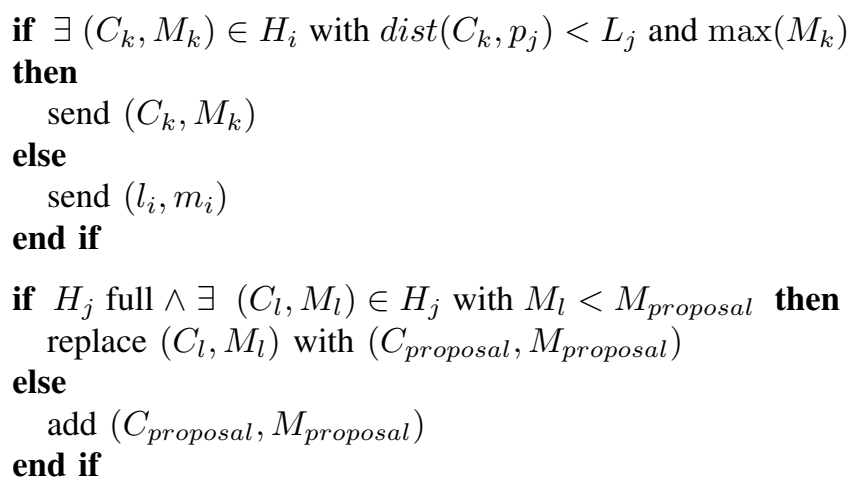

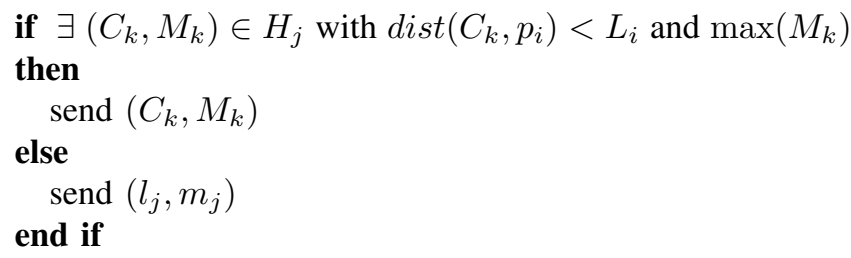

In order to identify nearby hot spots based on the local view of individual clients, we use this extended version of the epidemic aggregation protocol presented in [7]. It is known to work in network topologies without small cuts and also efficiently in dynamic network topologies. Here, range constraints have been additionally introduced to the maximum aggregation protocol, reflecting specifics of the locality in DDVEs. By allowing peers to verify that a hot spot's maximum center of mass lies within the random neighbor's lookahead range, only information from within the peer's lookahead is aggregated and hot spots transcending the lookahead range will be deleted from the local list. Furthermore, as only fixed-size information on hot spots is exchanged in periodic intervals, the bandwidth consumption of the detection algorithm is constant.

\section{Inverted Gravitation}

While detecting hot spots ideally happens as fast as possible, for their alleviation, i.e. the allocation of resources in response to this information, it is necessary to introduce some form of inertia as otherwise temporary surges or other systemdisturbing effects cannot be purposively absorbed.

In order to achieve such a stabilizing effect, we introduce the concept of inverted gravitation influencing the positions of virtual peers. For this, we assume that hot spots nearby a virtual peer $p_{i}$ exert the following force $F_{i}$ on its position:

$$
F_{i}=\frac{1}{G} \cdot \frac{m_{i} \cdot M_{i}}{r^{2}} .
$$

Formally following the previous notation with $m_{i}$ being the mass of a peer $p_{i}$ and $M_{i}$ the cummulative mass of the hot spot $h_{i}$, as well as $r$ denoting the distance betweeen the two point masses, this behavior is defined utilizing the inversion of the gravitational constant $G$. In other words, virtual peers change their positions and are drawn towards hot spots based on their mass, but unlike in the common definition of gravitation, the closer they are to their center of mass, the slower they become, i.e. the gravitational force is weakening.

To avoid a concentration of virtual peers on the very center of hot spots and thus provide a better coverage of the area the following adjustment step is taken:

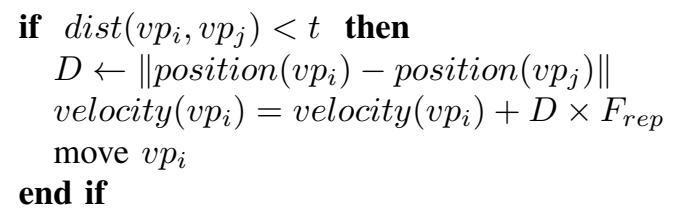




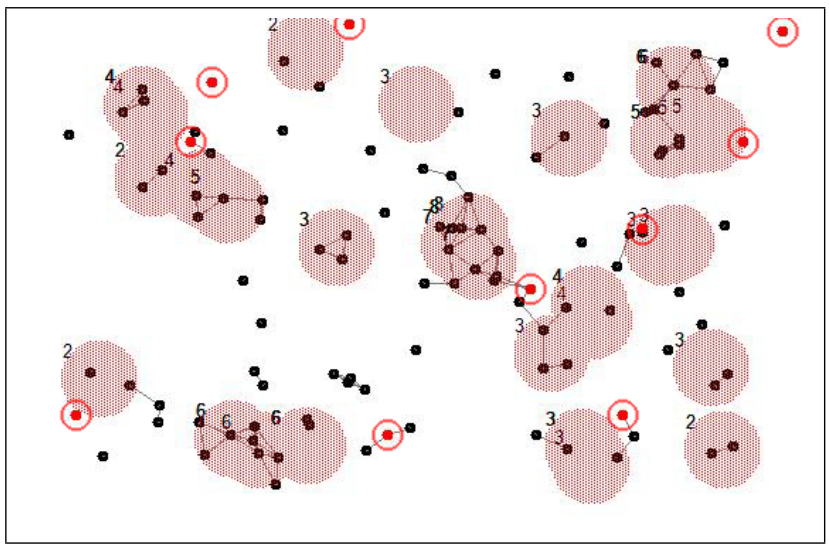

(a) Simulation Step 50

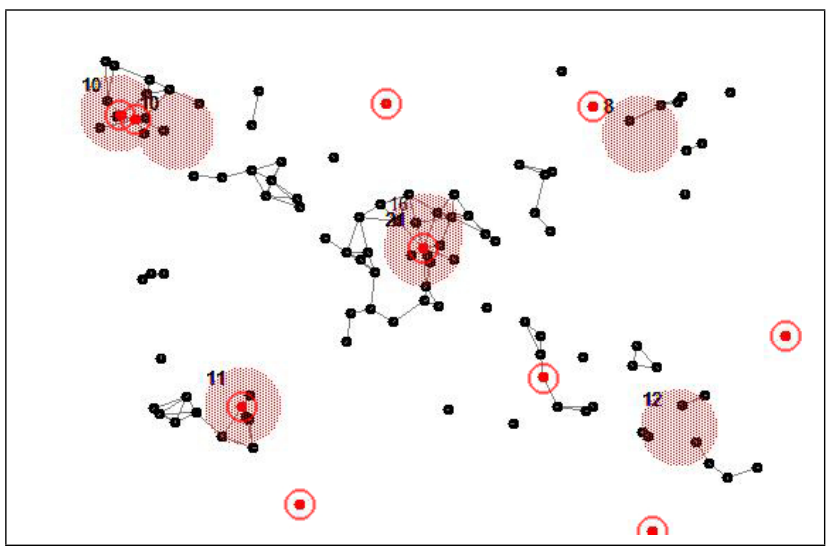

(c) Simulation Step 500

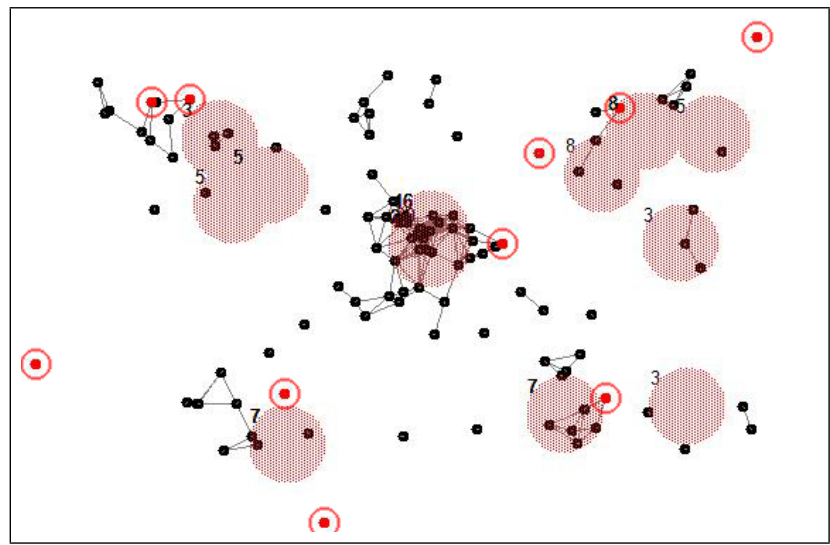

(b) Simulation Step 250

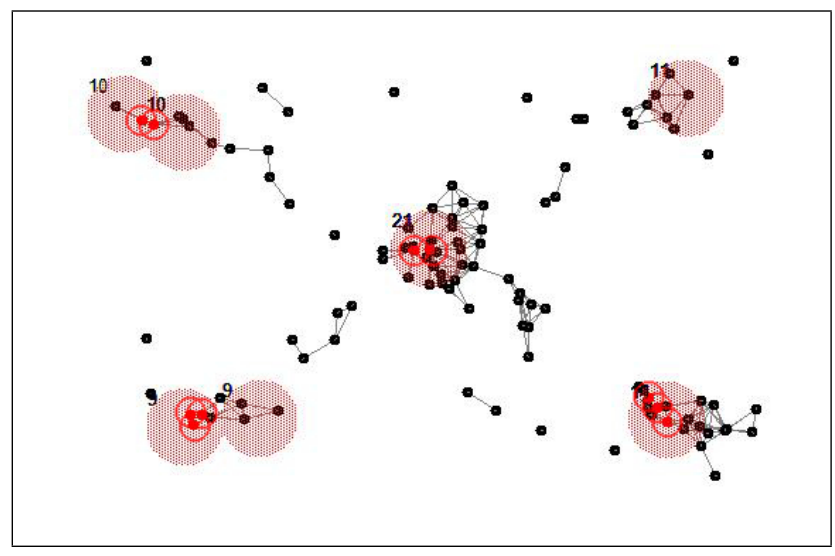

(d) Simulation Step 2500

Fig. 2. Snapshots of the resource allocation scheme in action (virtual peers are marked additionally to guide the eye)

Once a virtual peer $v p_{i}$ comes close to another virtual peer $v p_{j}$ within a threshold $t$, he is propelled away from the other in direction $D$ according to an additional repulsion constant $F_{\text {rep }}$ applied between two virtual peers. As a positive side effect of this improved coverage, also connections to the virtual peers will distribute more evenly.

Overall, the scheme creates the following pattern of action: After the detection of hot spot regions, the introduced inertia ensures that only relevant hot spots are covered subsequently by leaving the necessary time for example to the backbone as management layer to intervene first. The longer a hot spot lasts and the bigger its mass is, the more likely it will also be covered by virtual peers. Conversely, and metaphorically speaking, once covering a hot spot area, the virtual peers for a while behave like shadows left from the accumulation of peers. Being hosted by multiplexers that belong to the fraction of highly stable machines with long session times, this pays tribute to the locality of reference and is particulary benefitial to normal peers with high churn rates, which are then likely to find a virtual peer to connect to in those regions.

\section{Example}

Figure 2 contains a chronological series of snapshots taken from a simulation, serving as a showcase illustration of the resource allocation scheme. The whole visualization video is available online for download ${ }^{2}$.

The mobility of clients does not follow a uniform distribution that would lead to them moving randomly. Instead, as motivated in II-B, peers are more likely to move towards high user densities in their surroundings. For the definition of the hot spot distribution, we devised a graphical probability map where the hot spot location probability is encoded over the possibility area by greyscale values ranging from black (hot spot) to white (other). The map utilized here induces a hot spot distribution similar to the dots on a regular dice depicting the number five. Hot spot regions thus form over time towards the corners of the area as well as in its center. The larger, hatched circular areas show where the peers - at that specific discrete timestep of the simulation - suspect hot spot regions, i.e. their current aggregated local knowledge. The numbers to the upper left of these areas state the perceived mass of the

\footnotetext{
${ }^{2}$ http://mocca.uni.lu/resourceallocation/
} 
hot spot. Unlike normal peers and multiplexers, virtual peers are additionaly marked both in color and through an orbital border surrounding them.

Figure 2a depicts the initial situation where all peers began moving in the virtual environment after having been randomly distributed in the plain. So far, only little information has been exchanged and therefore peers according to their current local knowledge suspect hot spots to be mostly in their immediate surroundings. For this reason, at this stage no clear orientation can be observed yet of the virtual peers' movement. In Figure $2 \mathrm{~b}$, hot spots are becoming more and more pronounced, and the tendency towards consolidation after some gossiping about assumed hot spot locations is clearly visible. Virtual peers now gravitate towards locations of higher mass which at this point are still indistinct but identifiable large patches showing the currently aggregated information on hot spot locations. The other peers, including multiplexers, move towards locations of interest following the topology's underlying statistical model given by the probability map. The next snapshot in Figure $2 c$ already clearly shows both high-quality estimated hot spot regions as well as virtual peers gravitating towards those with the highest mass in proximity. A number of virtual peers have already taken position within the respective centers. Finally, Figure $2 \mathrm{~d}$ shows a later view of the particular situation. Hot spots and their masses have been detected almost perfectly, and due to their now statical character by having exposed enough stability, all virtual peers have concentrated within them.

\section{Evaluation}

In order to evaluate the resource allocation scheme, we conducted simulations utilizing the network topology generation and simulation environment TopGen [8]. Largely tailored to DDVEs, it provides different mobility models for a deterministic and event-based simulation of user movement. The model underlying the simulations resembles the "Preferential Attachment" scheme in the modelling of power law graphs [9], with the probability of a client to visit a certain point in the space being proportional to the number of users in that point's surrounding.

The simulated region constituting an excerpt of a DDVE scenario is sized 1000 x 650 pixels and populated with 100 peers and multiplexers hosting an additional 10 virtual peers. This is a conservative estimate fitting the ratio in terms of reliability and power of the multiplexers as described in detail in Section III-A.

The effective operation of the proposed resource allocation scheme is reflected in the accuracy development shown in Figure 3: After the initial mixing phase, on the one hand the hot spot detection takes place very fast due to the epidemic protocol. Already after around 300 steps a hot spot detection accuracy of more than $85 \%$ has been reached, which equals on average less than 2.5 exchanged messages per peer. Not reaching the optimal value of $100 \%$ as well as the slight fluctuation is due to the limitations by the utilized fixed-size lists that cause every now and then a known, correct hot spot to make way for another, heavier one. As a consequence,

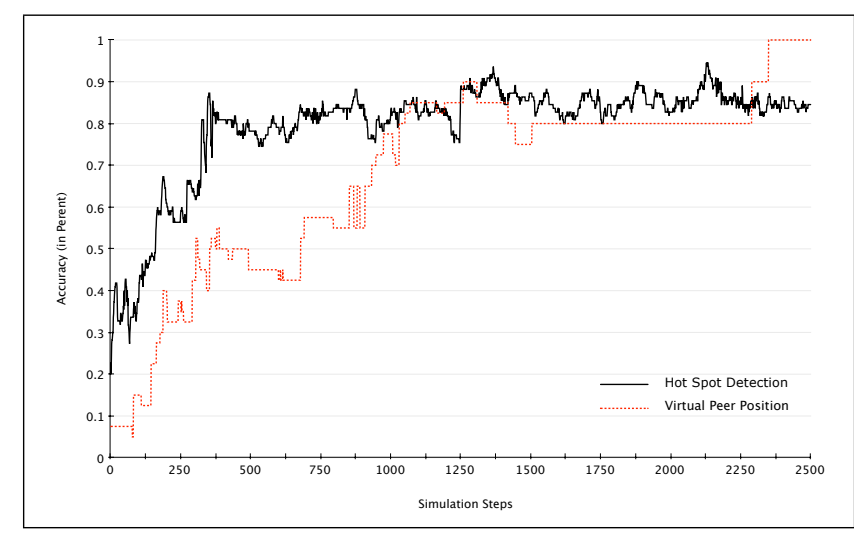

Fig. 3. Accuracy development

that knowledge is temporarily lost and will be aggregated back later. The lower gradient of the virtual peer position accuracy bears witness to the inertia introduced by the inverted gravitation concept. As desired, only when hot spots prove to be stable enough all virtual peers concentrate on them according to their mass. This happens here after circa 1000 steps, ultimately reaching an optimal coverage accuracy after all hotspots having been identified as relevant in terms of persistency.

Due to the epidemic scheme, all this moreover happens at a constant communication cost as depicted in Figure 4. The line in the plot has been added for reference and shows the average per-peer amount of messages exchanged (0.895) in one step. The exact value depends largely on the overlay density and the gossip frequency, but will nonetheless remain constant.

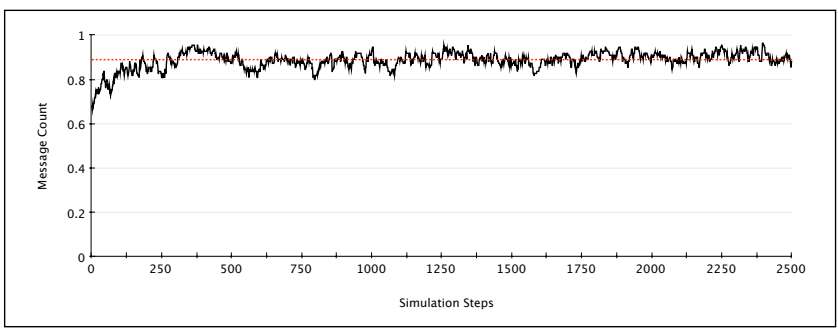

Fig. 4. Average communication cost

The node degree development depicted in Figure 5 showcases the effect of the preferential connect to virtual peers on the overlay. Once the positions of virtual peers reach a certain accuracy, i.e. are within or close to relevant hot spots, they level out the node degree to an average value of circa 2.4, leading to an overall sparser topology. Implicitly, this already unburdens the backbone, but as a direct consequence the node degree development not only supports the fast convergence of the detection scheme towards an optimal value but also ensures through stronger locality that peers have more knowledge on hot spots in their proximity than distant, less relevant ones.

This is an advantageous behavior of the scheme that is reflected in the range measure shown in Figure 6. Opposing 


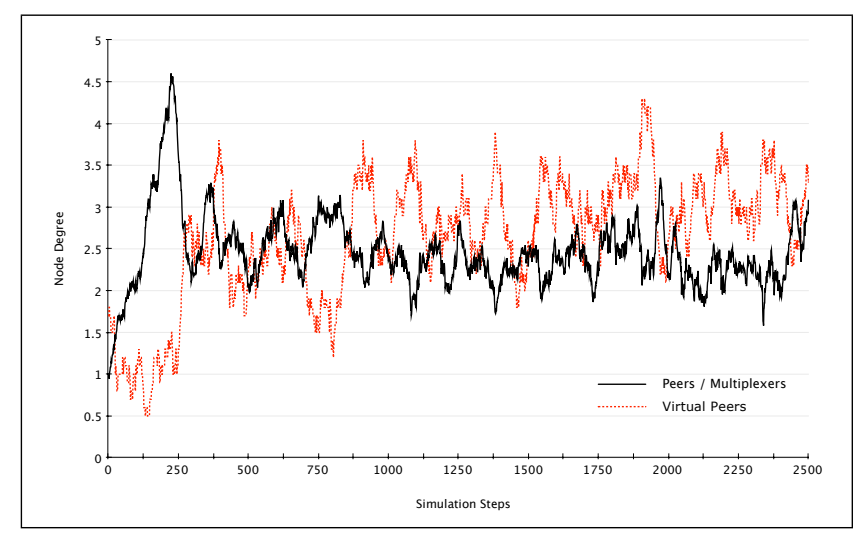

Fig. 5. Overlay node degree development

the average distance to known hot spot regions to unknown ones shows, that even after the initial mixing phase our scheme favors the discovery of nearby and thus more relevant hot spot regions.

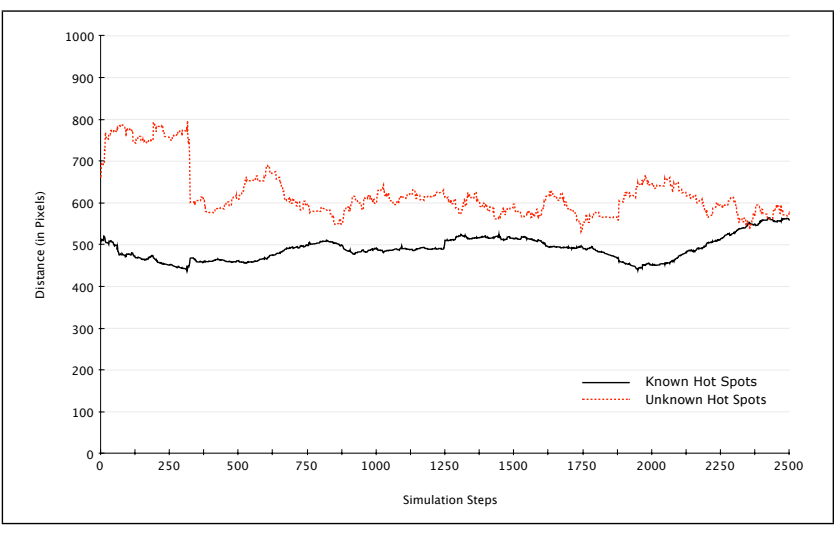

Fig. 6. Distance Ratio

Only when virtual peer positioning reaches $100 \%$, the average distance consequently converges to one value. This is due to the static nature of the hot spot distribution underlying the simulated scene leading to the knowledge on hot spot regions reaching stability. With the formation of other hot spot regions, again first the local ones would be favored and alleviated with the virtual peers taking the respective positions.

\section{RELATED WORK}

The issue of identifying and alleviating hot spots and, as their more dynamic variant, flash crowds occuring in distributed systems is a widely studied subject. In client-server settings, traditionally a detection would be followed - if at all - by measures usually entailing substantial loss of quality of service. For instance in [10] performance degradations are being monitored to detect hot spots, with the consequence being that no more users are admitted to the identified regions, resulting in a seriously impaired user experience. Later clientserver based approaches like [11] and [12] have already identified P2P technologies as complementary measure, when necessary utilizing P2P networks to unburden the inital centralized network while not substantially derogating the experience. P2P architectures specifically designed for the provision of DDVEs are for instance VON [13], Solipsis [14], FLoD [15] or Mediator [16]. While particularly the more recent approaches consider the heterogeneity of peers to allocate different roles to them within the network, they basically constitute a single P2P network which then is structurally rearranged to mitigate effects arising from hot spot regions. Common techniques are based on interest management, for instance the reduction of the size of the individual Area of Interest (AoI) as performed in the Voronoi-based clustering in [13], or the adjustment of AoI shapes in FLoD [15]. Also in [14], the mitigation involves immediate and more costly readjustments of the supporting network.

The idea to exploit heterogeneity in P2P networks in a similar way to cushion effects arising from hot spots can be mainly found in super-peer load balancing approaches. These superpeers as investigated e.g. in [17], [18] or [19] resemble the multiplexers in our scheme and take additional tasks according to their capability and then reshape the networks accordingly. In [18] the idea of so-called virtual servers is introduced, which are altruistically maintained data hosting blocks kept within peers. Each peer maintains at least one of these, and an algorithm constructs another overlay that efficiently distributes the virtual servers in a way that super-peers shoulder a large amount of the data to balance the load. Mainly in connection with spatial computing, some approaches introduce a distance measure for their protocols such as in [17]. Here, a proximityaware protocol based on a computed measure that utilizes the decentralized Vivaldi network coordinate system [20] to assign (5-dimensional, virtual coordinates) is proposed. Superpeer election is also handled in a decentralized fashion, and has also been considered in [21] for unstructured networks. The proposed $\mathrm{H}_{2} \mathrm{O}$ protocol is an interesting option in case that there is no reliable backbone facilitating super-peer election, but while mentioning a possible utilization of superpeers to allocate resources where needed, no concrete method is presented. Another super-peer approach operating on an unstructured network level is described in SOSPNet [19]. Load balancing is tackled here by automatically discovering semantic relationships between files to which pointers are maintained by the super-peers which form the second layer in a dual-layer network.

Most super-peer mechanims dealing with phenomena similar to hot spots have been considered in conjunction with file sharing scenarios, and thus do not consider specific properties of virtual environments influencing their formation like the inherent spatial aspect and characteristics of user mobility.

\section{Conclusion And Perspectives}

In this paper we have presented an efficient self-organized resource allocation scheme for P2P-based DDVEs, working with locally available information to identify and alleviate hot spot regions regardless of the underlying topology.

Exploiting the heterogeneity of clients, stronger peers partic- 
ularly in terms of bandwidth harness resources and contribute to data distribution by splitting off virtual peers. A gossipbased scheme is used to detect hot spots in virtual proximity while a gravitational field created from the aggregated local information guides the virtual peers to these regions.

In consequence of utilizing epidemic aggregation, local views converge fast to a global one while peer dynamics do not constitute a problem. Furthermore, the inertia introduced by the gravitational model for the virtual peers shows favorable structural effects.

We will discuss shortcomings of the presented approach in the following. Firstly, for the conducted simulations we utilized an artificial mobility model not involving churn explictly. Therefore it is planned to also perform simulations on the basis of real-world avatar traces from Second Life gathered at the University of Singapore [22]. This could yield further insights for the optimization of our scheme, for instance based on the measured global number of exchanged messages.

Secondly, depending on the specific situation, density and resulting connectivity, possible anomalies similar to localitybased failures occuring in greedy routing need to be further investigated. Despite being borderline situations, testing the behavior of the scheme in this regard would be useful. Another question that might arise is what happens to virtual peers stuck in between equal masses. So far, if that kind of situation occurs, eventually another virtual peer's repulsive force would be needed to release the stuck one. An extension of the scheme considering maximum force instead of maximum mass in these situations could for instance be an explicit solution here.

While being initially designed for the application in DDVEs, we believe that the resource allocation scheme presented here also opens up interesting applications for the monitoring of systems with dynamic nodes embedded into a geographic context in general.

\section{ACKNOWLEDGMENT}

The work presented in this paper was supported in part by the Fonds national de la Recherche (Luxembourg).

\section{REFERENCES}

[1] J. Botev, M. Esch, A. Höhfeld, H. Schloss, I. Scholtes, and P. Sturm, "The HyperVerse - concepts for a federated and torrent-based "3D web", International Journal of Advanced Media and Communication, vol. 2, no. 4, pp. 331-350, 2008.

[2] B. Cohen, "Incentives build robustness in BitTorrent," in Proc. 1st Workshop on the Economics of Peer-to-Peer Systems, Berkeley, CA, USA, 2003.

[3] D. Stutzbach and R. Rejaie, "Understanding churn in peer-to-peer networks," in Proc. 6th ACM SIGCOMM Conference on Internet Measurement (IMC '06), Rio de Janeiro, Brazil, 2006, pp. 189-202.

[4] M. Esch, J. Botev, H. Schloss, and I. Scholtes, "GP3 - a distributed grid-based spatial index infrastructure for massive multiuser virtual environments," in Proc. 14th IEEE International Conference on Parallel and Distributed Systems (ICPADS 2008), Melbourne, Australia, 2008, pp. 811-816.

[5] D. Kempe, A. Dobra, and J. Gehrke, "Gossip-based computation of aggregate information," in Proc. 44th Annual IEEE Symposium on Foundations of Computer Science (FOCS'03), Cambridge, MA, USA, 2003, pp. 482-491.
[6] F. Chung, Spectral Graph Theory. American Mathematical Society, 1997.

[7] M. Jelasity, A. Montresor, and O. Babaoglu, "Gossip-based aggregation in large dynamic networks," ACM Transactions on Computer Systems, vol. 23, no. 3, pp. 219-252, 2005.

[8] I. Scholtes, J. Botev, M. Esch, A. Höhfeld, H. Schloss, and B. Zech, "TopGen - internet router-level topology generation based on technology constraints," in Proc. 1st International Conference on Simulation Tools and Techniques for Communications, Networks and Systems (SIMUTools 2008), Marseille, France, 2008, pp. 1-10.

[9] A.-L. Barabsi and R. Albert, "Emergence of scaling in random networks," Science, vol. 286, no. 5439, pp. 509-512, 1999.

[10] X. Chen and J. S. Heidemann, "Flash crowd mitigation via adaptive admission control based on application-level observations," ACM Transactions on Internet Technology (TOIT), vol. 5, no. 3, pp. 532-569, 2005.

[11] C. Pan, M. Atajanov, M. B. Hossain, T. Shimokawa, and N. Yoshida, "FCAN: Flash crowds alleviation network," in Proc. 2006 ACM Symposium on Applied Computing (SAC), Dijon, France, 2006, pp. 759-765.

[12] V. N. Padmanabhan, H. J. Wang, P. A. Chou, and K. Sripanidkulchai, "Distributing streaming media content using cooperative networking," in Proc. 12th International Workshop on Network and Operating System Support for Digital Audio and Video (NOSSDAV 2002), Miami Beach, FL, USA, 2002, pp. 177-186.

[13] S.-Y. Hu, J.-F. Chen, and T.-H. Chen, "Von: A scalable peer-to-peer network for virtual environments," IEEE Network, vol. 20, no. 4, pp 22-31, 2006.

[14] D. Frey, J. Royan, R. Piegay, A.-M. Kermarrec, E. Anceaume, and F. L. Fessant, "Solipsis: A decentralized architecture for virtual environments," in Proc. 1st International Workshop on Massively Multiuser Virtual Environments (MMVE), Reno, Nevada, USA, 2008.

[15] S.-Y. Hu, T.-H. Huang, S.-C. Chang, W.-L. Sung, J.-R. Jiang, and B.-Y. Chen, "FLoD: A framework for peer-to-peer 3D streaming," in Proc. 27th Conference on Computer Communications (IEEE InfoCom '08), Phoenix, AZ, USA, 2008, pp. 1373-1381.

[16] L. Fan, H. Taylor, and P. Trinder, "Mediator: A design framework for P2P MMOGs," in Proc. 6th ACM SIGCOMM workshop on Network and system support for games, Melbourne, Australia, 2007, pp. 43-48.

[17] G. P. Jesi, A. Montresor, and O. Babaoglu, "Proximity-aware superpeer overlay topologies," IEEE Transactions on Network Science and Service Management, vol. 4, no. 2, pp. 74-83, 2007.

[18] S. Surana, B. Godfrey, K. Lakshminarayanan, R. Karp, and I. Stoica, "Load balancing in dynamic structured peer-to-peer systems," Performance Evaluation, vol. 63, no. 3, pp. 217-240, 2006.

[19] P. Garbacki, D. H. J. Epema, and M. van Steen, "Optimizing peer relationships in a super-peer network," in Proc. 27th International Conference on Distributed Computing Systems (ICDCS '07), Toronto, Ontario, Canada, 2007, p. 31.

[20] F. Dabek, R. Cox, F. Kaashoek, and R. Morris, "Vivaldi: A decentralized network coordinate system," ACM SIGCOMM Computer Communication Review, vol. 34, no. 4, pp. 15-26, 2004.

[21] V. Lo, D. Zhou, Y. Liu, C. Gauthierdickey, and J. Li, "Scalable supernode selection in peer-to-peer overlay networks," in Proc. Second International Workshop on Hot Topics in Peer-to-Peer Systems (HOT$P 2 P$ '05), La Jolla, CA, USA, 2005, pp. 18-27.

[22] H. Liang, R. N. Silva, W. T. Ooi, and M. Motani, "Avatar mobility in user-created networked virtual worlds: measurements, analysis, and implications," Multimedia Tools and Applications, vol. 45, no. 1-3, pp. 163-190, 2009. 